\title{
On packing density of growing size circles
}

\author{
Lajos László \\ Departement of Numerical Analysis, \\ Eötvös Loránd University, Budapest \\ email: laszlo@numanal.inf.elte.hu
}

\begin{abstract}
For any given natural number an arrangement of growing size circles, a packing of the plane will be constructed such that its packing density coincides - in the asymptotical sense - with that of the 'classical' hexagonal circle packing!
\end{abstract}

\section{The construction}

For a natural $n \geq 3$ let us define a special circle packing as follows. First, we circumscribe the unit circle with $n$ circles of the same radius $r_{n, 1}$ such that they also touch their both neighbours. Thus we get zone one, $Z_{n, 1}$.

Then we draw $n$ circles of the same radius $r_{n, 2}$ such that they touch two circles from $Z_{n, 1}$ and also their both neighbours with radius $r_{n, 2}$, getting this way $Z_{n, 2}$, etc.

Denote by $S_{n, k}$ the set of circles of the first $k$ zones:

$$
S_{n, k}=\cup_{i=1}^{k} Z_{n, i}
$$

and let

$$
S_{n}=\cup_{k=1}^{\infty} S_{n, k} .
$$

Then $S_{n}$ is a packing of the plane, an infinite set of circles with pairwise disjoint interiors, and a natural problem is to find the fraction of the plane filled by the circles making up this packing.

2010 Mathematics Subject Classification: 52C15, 52C26

Key words and phrases: circle packing, packing density 


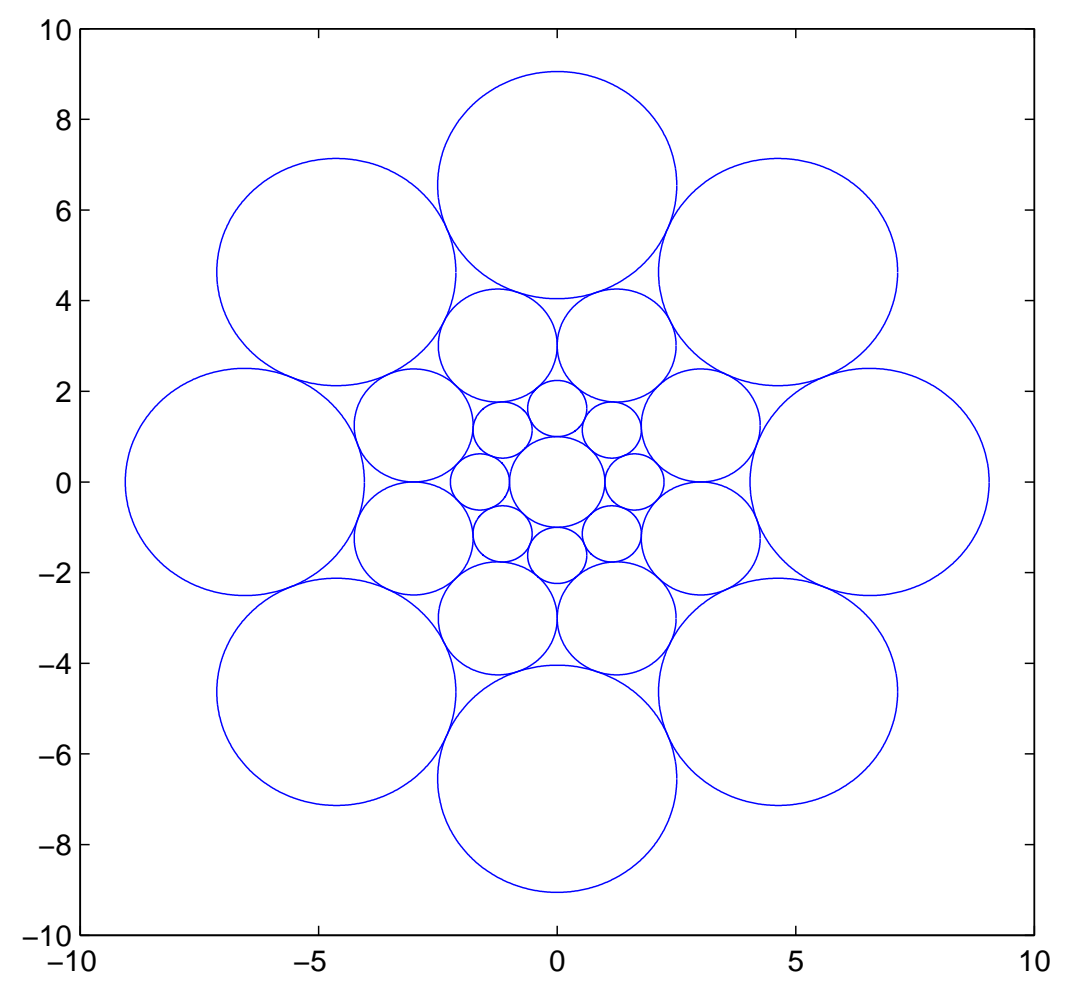

Figure 1. The set $S_{8,3}$, i.e. the first three zones for $n=8$.

\section{The main theorem}

The packing density of the arrangement $S_{n}$ related to a bounded domain $\mathrm{D} \subset \mathbb{R}^{2}$ is the ratio

$$
\frac{\sum|\mathrm{C} \cap \mathrm{D}|}{|\mathrm{D}|}, \mathrm{C} \in \mathrm{S}_{\mathrm{n}}
$$

where $|\cdot|$ denotes the area of its argument. It is customary to define (see e.g. Kuperberg [1]) the packing density in an Euclidean space by means of a limit, taking e.g. balls $B_{r}$ of radius $r$ centered at the origin:

$$
\lim _{r \rightarrow \infty} \frac{\sum\left|C \cap B_{r}\right|}{\left|B_{r}\right|}, C \in S_{n} .
$$


However, in our case taking polygons is more capable. Denote by $P_{n, k}$ the regular $n$-gon with vertices at the centres of circles in $Z_{n, k}$, and let

$$
\delta_{n, k}=\frac{\left|S_{n} \cap P_{n, k}\right|}{\left|P_{n, k}\right|} \equiv \frac{\left|S_{n, k} \cap P_{n, k}\right|}{\left|P_{n, k}\right|} .
$$

Then,

$$
\delta_{n}=\lim _{k \rightarrow \infty} \delta_{n, k}
$$

is the packing density of $S_{n}$, and

$$
\delta^{*}=\lim _{n \rightarrow \infty} \delta_{n}
$$

is the quantity we are interested in.

Theorem 1 With the notations above we have

$$
\delta^{*}=\frac{\pi}{2 \sqrt{3}} .
$$

Remark 1 As is known (see e.g. the survey on the first page in [2], showing the contributions of Lagrange, A. Thue, L. Fejes Tóth to the subject), the optimal packing density for circles is just this quantity - a curious coincidence!

Remark 2 The interested reader should also consult [3] and [4] for further information.

\section{The proof}

Let $n, k \in \mathbb{N}, n \geq 3$ be given. Assume that the centre of one of the circles belonging to $Z_{n, 1}$ lies on the $x$-axis, i.e. at $A_{n, 1}:=\left(1+r_{n, 1}, 0\right)$.

Denote by $B_{n, 1}$ the point, where the half-line $y=\tan \left(\frac{\pi}{n}\right) x$ is tangent to the circle chosen. It suffices to consider the 'basic' sector $\mathrm{B}_{n, 1} \mathrm{O} A_{n, 1}$, as is seen on Figure 2 for the case $n=8, k=3$.

It is easy to see that the centre $A_{n, i}$ of the $i$ - th circle in the basic sector lies on the $x$-axis for $i$ odd, and on the line $y=\tan \left(\frac{\pi}{n}\right) x$ for $i$ even, and just reversely for the $B_{n, i}^{\prime} s$. Also note that the angles at the $B_{n, i}^{\prime} s$ are rectangles. Introduce now the notations

$$
s_{n}=\sin \left(\frac{\pi}{n}\right), \quad t_{n}=\tan \left(\frac{\pi}{n}\right) .
$$




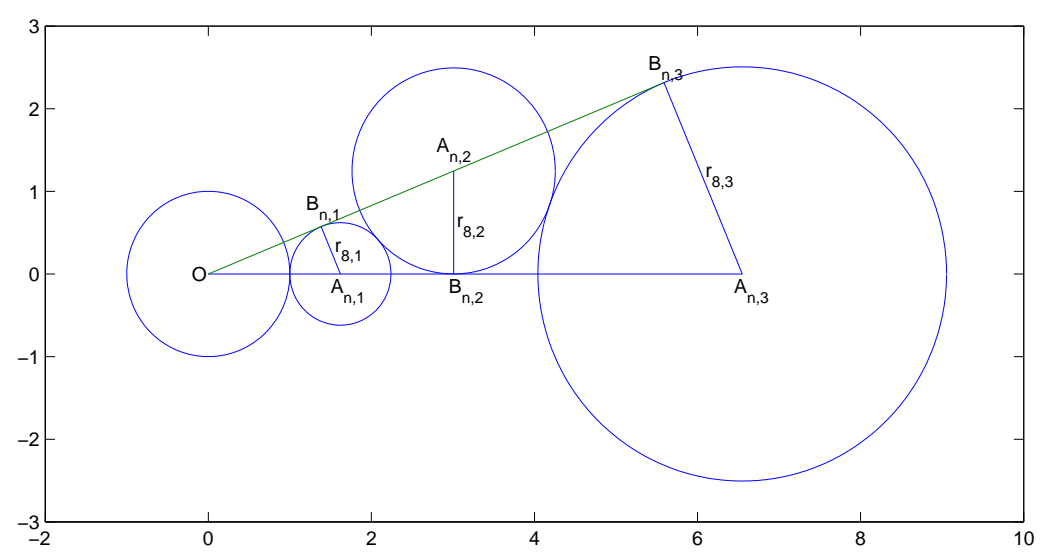

Figure 2. The basic sector for $S_{8,3}$

The radius $r_{n, 1}$ can be obtained from the triangle $B_{n, 1} O A_{n, 1}$ :

$$
s_{\mathrm{n}}=\frac{\mathrm{r}_{\mathrm{n}, 1}}{1+\mathrm{r}_{\mathrm{n}, 1}} \Rightarrow \mathrm{r}_{\mathrm{n}, 1}=\frac{\mathrm{s}_{\mathrm{n}}}{1-\mathrm{s}_{\mathrm{n}}}
$$

Since $A_{n, 1} B_{n, 2} A_{n, 2} B_{n, 1}$ and $A_{n, 2} B_{n, 3} A_{n, 3} B_{n, 2}$ are similar quadrilaterals (in fact, both are inscribed quadrilaterals with two rectangles), it follows that $r_{n, 2} / r_{n, 1}=r_{n, 3} / r_{n, 2}$, giving in general

$$
r_{n, k}=r_{n, 1} q_{n}^{k-1}, \quad q_{n}=\frac{r_{n, 2}}{r_{n, 1}} .
$$

The area of the polygon $P_{n, k}$ is $2 n$ times the area of triangle $O A_{n, k} B_{n, k}$, i.e.

$$
\left|P_{n, k}\right|=\frac{n}{t_{n}} r_{n, k}^{2}=\frac{n r_{n, 1}^{2}}{t_{n}} q_{n}^{2 k-2} .
$$

The sum of areas of the circles in $S_{n, k}$ is

$$
n \pi \sum_{i=1}^{k} r_{n, i}^{2}=n \pi r_{n, 1}^{2} \sum_{i=1}^{k} q_{n}^{2 k-2}=n \pi r_{n, 1}^{2} \frac{q_{n}^{2 k}-1}{q_{n}^{2}-1} .
$$

However, the contribution of the $k$-th zone to $\left|Z_{n, k} \cap P_{n, k}\right|$ is only $\frac{n-2}{2} \pi r_{n, k}^{2}$, instead of $n \pi r_{n, k}^{2}$. Consequently we have

$$
\left|S_{n, k} \cap P_{n, k}\right|=\pi r_{n, 1}^{2} q_{n}^{2 k-2}\left(\frac{n}{q_{n}^{2}-1}+\frac{n-2}{2}\right)-\frac{\pi n r_{n, 1}^{2}}{q_{n}^{2}-1} .
$$


When calculating the limit of $\delta_{n, k}$ for $k \rightarrow \infty$, the magnitude of the quotient $\mathrm{q}_{\mathrm{n}}=\frac{\mathrm{r}_{\mathrm{n}, 2}}{\mathrm{r}_{\mathrm{n}, 1}}$ is decisive. Introducing the new variable

$$
t=\tan \left(\frac{\pi}{2 n}\right)
$$

(cf. the standard trigonometric substitution $t=\tan \left(\frac{x}{2}\right)$ in calculus) we have

$$
s_{n}=\frac{2 t}{1+t^{2}}, \quad t_{n}=\frac{2 t}{1-t^{2}}
$$

and also

$$
r_{n, 1}=\frac{2 t}{(1-t)^{2}}, \quad r_{n, 2}=\frac{2 t(u+2 t \sqrt{v})}{(1+t)^{2}(1-t)^{4}}
$$

where

$$
u=1+4 t^{2}-t^{4}, \quad v=\left(3-t^{2}\right)\left(1+t^{2}\right) .
$$

The radius $r_{n, 2}$ can be calculated by considering the triangles $A_{n, 1} B_{n, 2} A_{n, 2}$ and $\mathrm{OB}_{n, 2} A_{n, 2}$. Analysing the function $t \rightarrow \frac{r_{n, 2}}{r_{n, 1}}$ we see that it is greater than one for $0<t<1$, or equivalently, that the relation $q_{n}>1$ holds for $n \geq 3$. Therefore, in case of $k \rightarrow \infty$ the second (negative) term in $\left|S_{n, k} \cap P_{n, k}\right|$ can be omitted to get

$$
\delta_{n}=\frac{\pi t_{n}}{r_{n, 2}^{2}-r_{n, 1}^{2}}\left(r_{n, 1}^{2}+\frac{n-2}{2 n}\left(r_{n, 2}^{2}-r_{n, 1}^{2}\right)\right)
$$

With the notation $\omega=\frac{n-2}{2 n}$ we obviously have $0<\omega<1$ for $n \geq 3$, which yields in a natural way the lower and upper bounds

$$
\delta_{n}^{0}<\delta_{n}<\delta_{n}^{1} .
$$

Since the difference of these bounds is simply

$$
\delta_{n}^{1}-\delta_{n}^{0}=\pi t_{n}=O(t) \quad(t \rightarrow 0),
$$

we can replace $\delta_{\mathfrak{n}}$ e.g. by its lower bound

$$
\delta_{n}^{0}=\frac{\pi t_{n} r_{n, 1}^{2}}{r_{n, 2}^{2}-r_{n, 1}^{2}}=\frac{\pi\left(1-t^{2}\right)^{3}}{2(2 t v+u \sqrt{v})}
$$

Having gotten rid of the singularity, we can immediately substitute $t=0$ (corresponding to $n \rightarrow \infty$ ) to get the desired result

$$
\delta^{*}=\lim _{n \rightarrow \infty} \delta_{n} .
$$

Considering this surprising coincidence, one puts the question: is there a more general principle, this conclusion can be drawn from? 


\section{References}

[1] W. Kuperberg, The set of packing and covering densities of convex disks, http://arxiv.org/pdf/1309.0281v1.pdf, (2013).

[2] Hai-Chau Chang, Lih-Chung Wang, A Simple Proof of Thues Theorem on Circle Packing, http://arxiv.org/pdf/1009.4322.pdf, (2010).

[3] K. Böröczky jr., Finite Packing and Covering, Cambridge University Press, (2004).

[4] C. A. Rogers, Packing and Covering, Cambridge, University Press, (1964).

Received: February 7, 2016 